\title{
Einleitung ins Thema «Die Zukunft unseres Lebensraumes - Eine Herausforderung an die Geographie»
}

Am 10. November 1989 organisierten die Geographisch-Ethnographische Gesellschaft Zürich sowie die Geographischen Institute der Universität und der Eidgenössischen Technischen Hochschule Zürich ein Symposium zum Thema «Die Zukunft unseres Lebensraumes - Eine Herausforderung an die Geographie». Diese Veranstaltung war Teil der Feiern zum 100-Jahr-Jubiläum der Geographisch-Ethnographischen Gesellschaft Zürich. Das Symposium war als fachlicher Teil der Geographie im Rahmen der 100-Jahr-Feiern für die Studierenden sowie Dozentinnen und Dozenten, aber auch für die Ehemaligen und die Öffentlichkeit bestimmt. Das Ziel war, an ausgewählten Beispielen die Stellung der Geographie in unserer Zeit und ihren Beitrag zur Lösung aktueller Probleme - im Sinne einer Standortbestimmung - herauszuarbeiten. Finanziell unterstützt wurde das Symposium durch die Geographische Kommission der Schweizerischen Akademie für Naturwissenschaften (SANW).

Im vorliegenden Heft der GEOGRAPHICA HELVETICA sind die schriftlichen Fassungen der vier Tagungsreferate von Bruno Messerli (Bern), Karl Stiglbauer (Wien), Gerhard Stiens (Bonn) und Peter Gresch (Zürich) wiedergegeben. Ergänzt werden diese Aufsätze durch die Festansprache «Gedanken zur Entwicklung der Zürcher Geographie in der 2. Hälfte unseres Jahrhunderts» von Gerhard Furrer (Zürich); diese wurden anläßlich der Jubiläumsfeier der Geographisch-Ethnographischen Gesellschaft Zürich am 30. September 1989 in Zürich gehalten.

Es ist keine Selbstverständlichkeit, daß die Vorträge einer wissenschaftlichen Tagung bereits wenige Wochen später in gedruckter Form vorliegen und so einem breiteren interessierten Publikum vorgestellt werden können. Dies war nur möglich dank des rechtzeitigen Eintreffens der Manuskripte und des großen Einsatzes der Redaktion der GEOGRAPHICA HELVETICA. Dafür sei den Autoren und den Redaktorinnen an dieser Stelle bestens gedankt.

\section{Der Lebensraum als Herausforderung?}

Viele sind am Lebensraum ${ }^{1}$ interessiert, wenige setzen sich dafür ein. Häufig wird - nicht zuletzt auch von der Raumplanung - bemängelt, daß der Lebensraum als Ganzes, im Gegensatz zu seinen einzelnen Elementen, über keine Lobby, über keine Anwälte verfüge. Das Engagement der Geographie zum Schutz und zur Erhaltung unseres Lebensraumes, aber auch zu seiner Gestaltung war bis anhin nicht allzu groß; darauf wird auch im Bericht «100 Jahre Geographisch-Ethnographische Gesellschaft Zürich» (JUD 1989, 143) hingewiesen. Zweifellos beschäftigte und beschäftigt sich die Geographie seit jeher und auf den verschiedensten Maßstabsebenen mit Problemen des Lebensraumes. Im Bericht «Geographie im Jahre 2000 - Forschungspolitische Früherkennung (FER) für die Geographie» (AERNI 1988) wird die umfassende problemorientierte Untersuchung der Landschaft als Lebensraum als Grundaufgabe der heutigen Geographie bezeichnet, und es wird u.a. gefordert, daß die $\mathrm{Zu}$ sammenhänge zwischen Gesellschaft und Lebensraum neu zu durchdenken seien. Ähnliche Aussagen finden sich auch im «Leitbild Geographie Schweiz» (LeIMGRUBer 1988). In Anlehnung an BOESCH (1986) ist allerdings festzuhalten, daß sich diese Auseinandersetzung bis anhin vor allem auf der deskriptiven und explikativen Ebene abspielte, während der normativen und operativen Ebene zu wenig Beachtung geschenkt wurde. "Auf der normativ-operativen Ebene geht es um die Gestaltung unseres Lebensraumes» (BOESCH 1986, 152). Dieses Normendefizit in der Geographie ist mit ein Grund, daß die Geographie von anderen Wissenschaften, gerade auch von solchen, welche sich ebenfalls mit Problemen unseres Lebensraumes auseinandersetzen, häufig noch als deskriptive «Verbreitungswissenschaft» angesehen wird, wodurch die interdisziplinäre Zusammenarbeit nicht erleichtert wird.

Die Auseinandersetzung mit den aktuellen Problemen unseres Lebensraumes, welche sich nicht allein auf die Beschreibung und Erklärung beschränken darf, sondern die Erarbeitung von Lösungsvorschlägen miteinschließen muß, ist eine stete Herausforderung, welcher sich die Geographie in Lehre, Forschung und Praxis zu stellen hat. Es muß gerade

Hans Elsasser, Prof., Dr., Geographisches Institut der Universität Zürich, Winterthurerstr. 190, 8057 Zürich 
auch den Vertretern unserer Disziplin zu denken geben, wenn vom Schweizerischen Wissenschaftsrat folgendes festgehalten wird: «Die Bedrohung der natürlichen Umwelt ist innerhalb weniger Jahre an die vorderste Stelle des öffentlichen Bewußtseins gerückt. Es verdient hier festgehalten zu werden, $\mathrm{da} ß$ dieser Bewußtseinswandel, abgesehen von einigen wenigen eindringlichen Mahnern - wozu sicherlich die Zürcher Geographen WINKLER, GUTERSOHN und EGLI zu zählen sind (Ergänzung H.E.) - nicht von der Fachwelt, sondern von der Öffentlichkeit selber, im besonderen den Umweltorganisationen, ausgegangen ist» (Schweizerischer Wissenschaftsrat 1989, 35).

\section{Die Zukunft als Herausforderung?}

Stellt die Auseinandersetzung mit der Zukunft eine Herausforderung an die Geographie dar? Daß sich die Geographie mit Fragen der Zukunft schwertat, belegt folgendes aus den siebziger Jahren stammende Zitat aus einem von einem Geographen verfaßten kleineren Lehrbuch der Landesplanung: «Innerhalb eines Vergleichs von Geographie und Landesplanung ist ein wesentlicher Aspekt auch das unterschiedliche Verhältnis zur Zukunft. Der Geograph ist, so sehr er auch einen geschärften Blick für künftige Entwicklungen haben mag, in seiner $\mathrm{Ar}$ beit letztlich gegenwartsbezogen. Das vorausschauende aktiv-gestaltende Element gehört dagegen auf Grund der gesellschaftspolitischen Verantwortung zum Wesensinhalt der Landesplanung. Entsprechendes gilt für das Problem der Prognose. Es ist unmöglich, Vorhersagen im menschlichen Bereich nach exakt wissenschaftlichen Methoden aufzustellen. Angesichts ihres im Grunde nicht wissenschaftlichen Charakters kann die Prognose daher nicht Bestandteil irgendeiner Wissenschaft sein, somit auch nicht Bestandteil der Geographie» (GILDEMEISTER 1973, 48).

Eine solche Haltung ist überholt. Der Schweizerische Wissenschaftsrat hält in seinem Bericht «Forschungsplatz Schweiz - Horizont 1995» fest: «Die Aufgabe der Umweltforschung besteht zunächst darin, aktuelle Probleme zu erkennen und ihre Ursachen zu ergründen. Heute steht fest, daß dies nicht genügt, sondern daß der Blick vermehrt in die Zukunft gerichtet werden muß. Nur so können Entwicklungen erkannt und beeinflußt werden, die jetzt bereits im Gang sind, deren Auswirkungen aber erst später zu spüren sein werden» (Scheizerischer Wissenschaftsrat 1989, 36). Im Aufsatz von STIENS in diesem Heft wird von der «Vergegenwärtigung von Zukunft» gesprochen.

Zum Glück geht es heute auch in der Geographie nicht mehr so sehr um die Frage, ob sie sich überhaupt mit der Zukunft auseinandersetzen soll und darf, sondern es stellen sich Fragen über die Methoden der Beschäftigung mit der Zukunft. Besonders beliebt sind gegenwärtig - nicht nur in der Geographie - Szenarien ${ }^{2}$. «Das Arbeiten mit Szenarien ist in vielen Bereichen - von der militärischen bis zur zivilen Planung, von der Politikberatung bis zur Forschung und wissenschaftlichen Früherkennung - ein Verfahren zur kreativen und spekulativen Auseinandersetzung mit der Zukunft geworden: Kreativ und spekulativ insofern, als der Vorstellungswelt relativ freie Zügel gelassen werden, ohne daß der Spielraum möglicher Wirklichkeiten völlig verlassen wird» (FREY, GÜLLER u. a. 1989, 0-6).

\section{Die Zukunft des Lebensraumes als Herausforderung?}

Als echte Herausforderung an die Geographie muß nun die Auseinandersetzung mit der Zukunft unseres Lebensraumes, z. B. die Erarbeitung von entsprechenden Szenarien, angesprochen werden. Der Raumbezug vieler Szenarien ist - zumindest in der Schweiz - eher gering. Dies erstaunt nicht, denn «eine räumliche Differenzierung allgemeiner $\mathrm{Zu}$ kunftsaussagen - also deren Projektion in ein korrektes räumliches Bezugsraster hinein - scheint nach allen bisherigen Erfahrungen das intellektuell Aufwendigste bei raumbezogenen Szenarien zu sein» (sTIENs 1982, 68). Wie sieht die Raumstruktur der Schweiz aus, wenn sich die vom Bundesrat im BERICHT ÜBER DIE LEGISLATURPERIODE 1987-1991 formulierte Leitidee des qualitativen Wachstums durchsetzen würde? Oder: Wie sieht die Raumstruktur der Schweiz Ende des 20. Jahrhunderts aus, wenn sich die Ziele des EG-Binnenmarktes durchgesetzt haben? Oder: Welche Auswirkungen auf die Raumstrukturen der Schweiz besitzen globale Klimaveränderungen?

Das Fehlen raumbezogener Szenarien, Konzepte, Leitbilder $^{3}$ und der geringe Raumbezug sektoraler Zukunftsaussagen wird gerade auch in der Raumplanung und Raumordnungspolitik auf allen Maßstabsebenen immer mehr als eine Schwachstelle erkannt ${ }^{4}$. Die Auseinandersetzung mit der Zukunft unseres Lebensraumes könnte deshalb der Raumplanung in der Schweiz neue und dringend notwendige Impulse verleihen.

Eine zukunftsorientierte Geographie kann deshalb ganz wesentlich beitragen zur Problemlösung, Gestaltung und Erhaltung unseres Lebensraumes. Wenn es der Geographie gelingt, durch Untersuchungen über die Zukunft unseres Lebensraumes dafür zu sorgen, daß dieser verantwortungvoller genutzt und gestaltet wird, hat sie auch den Leistungsausweis einer ganzen Disziplin erbracht. Verantwortungsvoll bedeutet auch, daß Freiräume für künftige Generationen erhalten und geschaffen werden, d. h. daß die Zukunft möglichst offen gehalten wird 
und daß man nicht der Versuchung erliegt, zu wissen, welches die «richtige» Lebensraumgestaltung auf alle Zeiten sein wird.

Zum Schluß sei der berühmte französische Paläontologe, Philosoph und Theologe PIERRE TEILHARD DE CHARDIN zitiert:

Die Erforschung der Vergangenheit und des Raumes an sich ist leer und enttäuschend, denn die wahre Wissenschaft ist die Wissenschaft der Zukunft, die nach und nach durch das Leben verwirklicht wird.

\section{Anmerkungen}

1 Unter Lebensraum wird der gesamte Raum, auf dem sich das Dasein des Menschen abspielt, verstanden. Der Begriff "Lebensraum» wird nicht in einem (verhängnisvollen) geopolitischen Sinn verwendet.

2 Beispielsweise bilden Szenarien wichtige Grundlagen für die laufenden Arbeiten in den beiden Nationalen Forschungsprogrammen "Boden" und "Stadt und Verkehr". Auch im Nationalen Forschungsprogramm "Regionalprobleme" sowie im ETH-Projekt MANTO oder bei der wissenschaftlichen Begleituntersuchung über Telekommunikations-Modellgemeinden (WBU KMG) wurde und wird mit Szenarien gearbeitet.

${ }^{3}$ Auch wenn hinter diesen Begriffen unterschiedliche Inhalte stehen, ist es eher von sekundärer Bedeutung, wie solche raumbezogenen Zukunftsaussagen genannt werden.

${ }^{4} \mathrm{Vgl}$. dazu den Raumplanungsbericht 1987.

\section{Literatur}

AERNI, K. (Red.) (1988): Geographie im Jahre 2000 - Forschungspolitische Früherkennung (FER) für die Geographie. Geographica Helvetica Nr. 1, 37-44.

BOESCH, M. (1986): Schweizer Geographie am Wendepunkt - Überlegungen zu einer Normativen Metatheorie. Geographica Helvetica Nr. 3, 147-154.

FREY, R.L., GÜLLER, P. u a. (1989): Szenarien der Stadtund Verkehrsentwicklung = Bericht 1 des NFP «Stadt und Verkehr», Zürich.

GILDEMEISTER, R. (1973): Landesplanung = Das Geographische Seminar, Braunschweig.

JUD, P. (1989): 100 Jahre Geographisch-Ethnographische Gesellschaft Zürich. Geographica Helvetica Nr. 3, 113-151.

LEIMGRUBER, W. (Red.) (1988): Leitbild Geographie Schweiz. Geographica Helvetica Nr. 1, 33-36.

SCHWEIZERISCHER BUNDESRAT: Bericht über den Stand und die Entwicklung der Bodennutzung in der Schweiz (Raumplanungsbericht 1987), Bern.

SCHWEIZERISCHER BUNDESRAT: Bericht über die Legislaturperiode 1987-1991 vom 18. Januar 1988, Bern.

SCHWEIZERISCHER WISSENSCHAFTSRAT (1989): Forschungsplatz Schweiz - Horizont 1995. Ziele für die schweizerische Forschungspolitik, Vorschläge des Wissenschaftsrates an den Bundesrat, Planungsperiode 1992-1995. Teil I: Ziele, Bern

STIENS, G. (1982): Zur Methodik und zu den Ergebnissen raumbezogener Szenarien - Erfahrungsbericht aus der $\mathrm{BRD}=$ Arbeitsbericht Nr. 30 des NFP "Regionalprobleme», Bern. 